\title{
FROTTEMENT INTÉRIEUR ET MICRODÉFORMATION DANS LE FER CHARGÉ EN HYDROGÈNE
}

\author{
J. San Juan, G. Fantozzi, C. Esnouf, F. Vanoni* et A. Bernalte** \\ Groupe d'Etudes de Métallurgie Physique et de Physique des Matériaux \\ (LA 341), I.N.S.A., Bât. 502, 69621 VizZeurbanne Cedex, France \\ ${ }^{*}$ C.E.N. Grenoble, D.R.F., 85 X, 38041 Grenoble Cedex, France \\ **Dpto Electricidad y Magnetismo U.N.E.D., Apdo 50487 Madrid, Espagne
}

\begin{abstract}
Résumé - iNous avons êtudié le comportement du pic de Snoek-Köster dû à Thydrogène dans le fer pur. Les deux composantes haute température et basse température du pic sont attribuées à la création de doubles décrochements sur les dislocations non-vis contrôlê par la diffusion des atomes ou des amas d'hydrogène.
\end{abstract}

Abstract - The beaviour of the Snoek-Köster peak due to hydrogen in pure iron has been studied. The two components of the peak at high and low temperatures are attributed to the double-kink generation on non-screw dislocations in the presence of core mobile hydrogen atoms or clusters.

\section{I - INTRODUCTION}

L'article de revue de Hirth (1) en 1980 pose bien le problème du pic de Snoek-Köster dû á $l^{\prime}$ hydrogène $5-K(H)$ dans le fer. Les résultats de frottement intérieur obtenus jusqu'à cette période (2-5), montraient un seul pic complémentaire du pic $\alpha$, qui se déplaçait foriement vers les basses températures avec la température de recuit. Pourtant, les ênergies d'activation mesurées étaient distribuées autour de deux valeurs : $0.21 \mathrm{eV}$ et $0.32 \mathrm{eV}$.

Cette dispersion apparente des énergies d'activation est maintenant comprise: en effet, des résultats récents (6-9), montrent 1 'existence de deux composantes du pic $\mathrm{S}-\mathrm{K}(\mathrm{H})$; une forte hydrogënation favorisant la composante haute température (HT) par rapport à la composante basse température $(B T),(6,8)$.

\section{II - METHODE EXPERIMENTALE}

Les expériences sont réalisées sur des plaquettes $(0.5 \times 5 \times 40 \mathrm{~mm})$ de Fer de haute pureté obtenu au C.E.N.G., recuit $10 \mathrm{~h}$ à $400^{\circ} \mathrm{C}$ sous hydrogène purifié (10). Un champ magnêtique axial de 200 De est appliqué de façon à éliminer les effets du couplage magnêto-élastique.

Tous les échantịlions sont préaíablement bien dêformēs à température ambiante de $2 \%$ en traction plus $2 \%$ en torsion bidirectionnelle. Ces deux déformations permettent d'éliminer le pic $\boldsymbol{\beta}_{\alpha}$ de frottement intérieur (11) et de créer les sites de piëgage de $T$ 'hydrogène lors de $T$ 'hydrogênation. Celle-ci est rëalisée par électrolyse ã température ambiante $\left(5 \%\right.$ de $\left.\mathrm{SO}_{4} \mathrm{H}_{2}+\mathrm{CS}_{2}\right)$ pendant 15 minutes avec un courant de $400 \mathrm{~mA}$.

Les mesures de frottement intérieur sont réalisées à l'aide d'un pendule de torsion inversé équipe $d^{3} u n$ microordinateur de table du type C.B.M. commodore 4032 (12).

\section{III - RESULTATS EXPERIMENTAUX}

Fer après hydrogénation

La Fig. I montre les spectres de frottement intérieur pour trois fréquences de sollicitation $(1.2,2.4$ et $4.2 \mathrm{hz})$, ainsi que 1 a courbe de variation du module correspondant à la fréquence intermëdiaire ; dans tous les cas l'amplitude de sol- 
licitation est égale à $\varepsilon_{m}=5 \times 10^{-6}$. On peut observer clairement le décalage vers les hautes températures pour les deux composantes du pic $S-K(H)$. Ce décalage est également mis en évidence après soustraction du fond continu selon une méthode décrite par ailleurs (13). Etant donné que la hauteur des deux composantes est indépendante de 1 a fréquence (Fig. 2), nous avons pu dëduire les énergies d'activation à partir du décalage moyen en tempêrature du flanc basse température du spectre pour 1 a composante B.T. et du flanc haute température pour la composante H.T. Nous avons ainsi obtenu pour la composante B.T. : $E_{B T}=0.21 \pm 0.02 \mathrm{eV}$; et pour la composante H.T. : $E_{H T}=0.38 \pm 0.03 \mathrm{eV}$.

Nous avons étudiē l'effet de 1 'amplitude de sollicitation sur les deux composantes du pic $S-K(H),(F i g .3)$. Les resultats que nous avons obtenu prêcédemment ( 8 ) sont plus nettement confirmes : La composante B.T. est indépendante de l'amplitude de sollicitation, tandis que la composante H.T. dépend fortement de l'amplitude de vibration. On peut remarquer également 1 'augmentation du fond continu à très basse température avec l'amplitude de vibration.

Fer hydrogêné et déformé à $85 \mathrm{~K}$

Nous avons étudié l'effet d'une déformation à basse tempērature sur le pic de $S-K(H)$, et l'évolution du spectre avec la tempêrature de recuit. La Fig. 4 montre le spectre de frottement intérieur imméaiatement aprēs déformation de $\pm 1 \%$ en torsion à $85 \mathrm{~K}$ (courbe 2). On peut remarquer la diminution de la hauteur des deux composantes ainsi que son élargissement du côté basses et hautes températures par rapport au spectre aprēs hydrogénation (courbe 1 ). Un recuit à $240 \mathrm{~K}$ (courbe 3 ) fait disparaître 1 'êlargissement basse température du pic et développe préféréntiellement la composante H.T.

L'effet des recuits ultérieurs est indiquẽ par la Fig. 5. La composante H.T. du pic $S-K(H)$ atteint son maximum pour des températures de recuit comprises entre $240 \mathrm{~K}$ et $260 \mathrm{~K}$. Le pic $\gamma$ situé vers $290 \mathrm{~K}$ présente une hauteur maximale lors de $1 \mathrm{a}$ première montée en temperature, comme cela a èté observé pour des échantilions non hydrogénés (9). Pour des recuits au-dessus de $290 \mathrm{~K}$, nous observons une rapide disparition de $\mathrm{Ta}$ composante $H . T$. du pic $\mathrm{S}-\mathrm{K}(\mathrm{H})$, une disparition moins rapide de $\mathrm{la}$ composante $B . T$. et la disparition du pic $\gamma$. Un recuit à $350 \mathrm{~K}$ élimine complètement toutes ces manifestations anélastiques.

\section{IV - DISCUSSION}

La relaxation $S-K(H)$ est généralement attribuée à $T$ 'interaction dislocationhydrogêne. La première interprétation proposée par Schoeck (14) attribue le pic S-K au trainage des atomes d'hydrogène par les dislocations selon le modèie de la corde víbrante. Plus récemment, Seeger (15) et Hirth (1) ont proposế indépendamment un modèle basê sur la création de doubles décrochements en prësence d'interstitiels d'hydrogène mobiles.

La présence de deux composantes constituant le pic $S-K(H)$ rend plus complexe son interprétation. La composante BT a été attribuêe par la plupart des auteurs (5-7) à $7^{\prime}$ interaction des dislocations non-vis $\left(71^{\circ}\right)$ avec les atomies d'hydrogëne, certains auteurs utilisant le modèle de la corde vibrante, d'autres le modèle des décrochements.

La composante HT a été attribuée par Vetter et al. (16) et Kê et al. (6) au mouvementdes décrochements géométriques sur les dislocations vis contrôté par le trainage des atomes d'hydrogène. D'autre part, Sakamoto et Shimada (7) attribuent cette composante à l'interaction des dislocations non-vis avec des amas d'hydrogène. Enfin, Hirth (1) propose pour la composante HT la crëation de doubles décrochements sur les dislocations vis en présence d'atomes d'hydrogène dans le coeur de la dislocation.

Dans le cas de 1 'hydrogène dans le fer, le môdèle de Schoeck, prēdit un volume d'activation de 1 'ordre de quelques centaines de $b^{3}$. Or, des expériences de microdéformation donnent un volume d'activation de l'ordre de 10 b3 pour les deux composantes du $S-K(H),(8)$. D'autre part, nous avons également montré que les deux composantes sont dues à 1 'interaction des dislocations non vis avec $T$ 'hydrogène. En effet, pour une forte hydrogénation, seule la composante HT apparait ; si cette composante est aue aux disiocations vis, les dislocations non-vis devraient donner 
lieu soit au pic $\propto$ soità la composante BT, ce qui n'est pas le cas. Les résultats de la Fig. 5 confirment d'ailleurs cette hypothèse : en effet, nous pouvons observer que la hauteur du pic $\gamma$ est i ndépendante de 1 'hydrogënation. Les mémes résul tats ont été obtenus par Matsui et Schultz (17). On peut donc exclure 1 'interprëtation de Hirth pour la composante H.T.

D'autre part, en ce qui concerne le modèle du mouvement des dēcrochements gẻométriques, les expêriences réalisées avec l'application d'une contrainte statique (8) montrent que cette application développe la composante H.T., alors que l'effet inverse devrait être observé (18). Par conséquent le modèle ne peut convenir pour interpréter la composante H.T.

Enfin, Seeger (19) a proposè un modèle du pic de S-K qui fait apparaître deux pics suivant la densité des dêcrochements, ayant une différence d'énergie d'activation de l'ordre de 7 'ënergie de crêation d'un dêcrochement. Or la diffërence observée expérimentalement est beaucoup plus grande que celle prévue par le modèle, qui ne peut donc être retenu.

Nous pouvons ainsi proposer l'interprétation suivante :

* La composante BT du pic S-K(H) dans le fer est due à la creation thermiquement activée de doubles décrochements sur les dislocations non-vis contrôlée par $1 a$ diffusion des atomes d'hydrogène sur la ligne de la dislocation.

* La composante HT est due au même processus mais contrôlé par la diffusion de petits amas d'hydrogène Hc (probablement de nature diatomique).

En effet, le volume d'activation mesuré pour les deux composantes est en accord avec le processus de crēation des doubles décrochements (18). Le fait d'observer une dépenaence plus torie avec l'amplitude de vibration de la composante HT peut être expliqué deĩa maniere suivante. Á basse température, les amas d'hydrogène restent immobiles et la longueur active resporisable de la composante BT est la distance LHc entre amas. Par contre à haute température, les amas d'hydrogène deviennent mobiles et la longueur active pour la composante HT est alors égale a la distance $L_{N}$ entre points d'ancrage durs (rig. 6). Cornme $L_{N} \gg L_{H C}$, la composante HT sera plus sensible à la contrainte appliquée alternative ou statique.

D'autre part, une déformation à basse température produit un désancrage aussi bien des atomes d'hydrogêne que des amas, provoquant une diminution de la hauteur des deux composantes. un recurt à $240 \mathrm{~K}$ permet. la diffusion de 1 'hydrogène vers les dislocations en favorisant la formation des amas et développant ainsi la composante HT (fig. 4). Des recuits à plus haute température dissocient d'abord les amas d'hydrogëne, puis provoquent la disparition de l'hydrogène par diffusion. Ainsi, la composante HT disparait avant la composante BT (Fig. 5).

Enfin, l'élargissement du côtê basse température du pic après déformation plastique à $85 \mathrm{~K}$ peut être attribué à un processus d'interaction entre les atomes d'hydrogène et les défauts ponctuels du type autointerstitiel créés lors de la déformation plastique. De plus, l'élargissement cóte haute température et son recuit préfërentiel (Fig. 4 et 5 ) est tout à fait compatible âvec le modèle des doubles décrochements (18).

\section{REFERENCES}

(1) Hirth J.P. : Met. Trans. A : 11A, p. 861 (1980)

(2) Gibala R. : Acta Met. 15, p. 428 (1967).

(3) lakıta K., Sakamoto K. : Scripta Met. 10, p. 399 (1976).

(4) Kronmüller H., Steeb H., König N. : II Nuovo Cimento 33B, p. 205 (1976).

(5) Ritchie I.G., Dufresne J.F., Moser P.: Phys. Stat. SoT. (a) 52, p. 331 (1979),

(6) Kè T.S., Kronmuli Ter H., Seeger A., Suu Z.0. : Hydrogen Effects in Metals (ed. by I.M. Bernstein, A.W. Thompson) The Met. Soc. of AIME (1981), p. 281

(7) Sakamoto K., Shimada M. : ICIFUAS-7, Juurnal de Pnysique C5, 42, p. 109 (1981).

(8) San Juan J., Fantozzi G., tsnouf C., vanoni F. : Proc. "Hydrogène et Matériaux $\mathrm{H}-3$ " (ed. P. Azou) Paris (1982) vol. 1, B-8.

(9) Dufresne J.F. : Thèse Université de Grenoble (1982)

(10) Vanoni F. : Thèse Universitê de Grenoble (1973). 
(11) San Juan J., No' L., Remon A., Esnouf C., Fantozzi G., Bernalt A. Anales de Fisica A: $79, \mathrm{D}, 34$ (1983).

(12) Bouvier-Volalil le J.L., San Juan J. : cette confërence

(13) No' L., San Juan J., Esnouf C., Fantozzı G., Bernalte A. : cette conférence

(14) Schoeck G. : Acta Met. 11 p. 617 (1963).

(15) Seeger A. : Phys. Stat. Sol. (a) 55 p. 457 (1979).

(16) Vetter K., Steeb H., Kronmull ler H. : Proc. "Hydrogène dans les Métaux H-2" Paris (19/7), 2 C5.

(17) Matsui H., Schultz H. : ICIFUAS-7, Journal de Physique C5, 42 p. 115 (1981).

(18) Fantozzi G., Esnouf C., Benoit W., Ritcnie I.G., : Progress in Materials Science, 27, p. 311 (1982).

(19) Seeger A. : Scripta Met., 16, p. 241 (1982).

\section{REMERCIEMENTS}

J. San Juan remercie le Dpto de Fisica del Estado Solido de Ta Universidad del Pais Vasco pour son soutien financier.
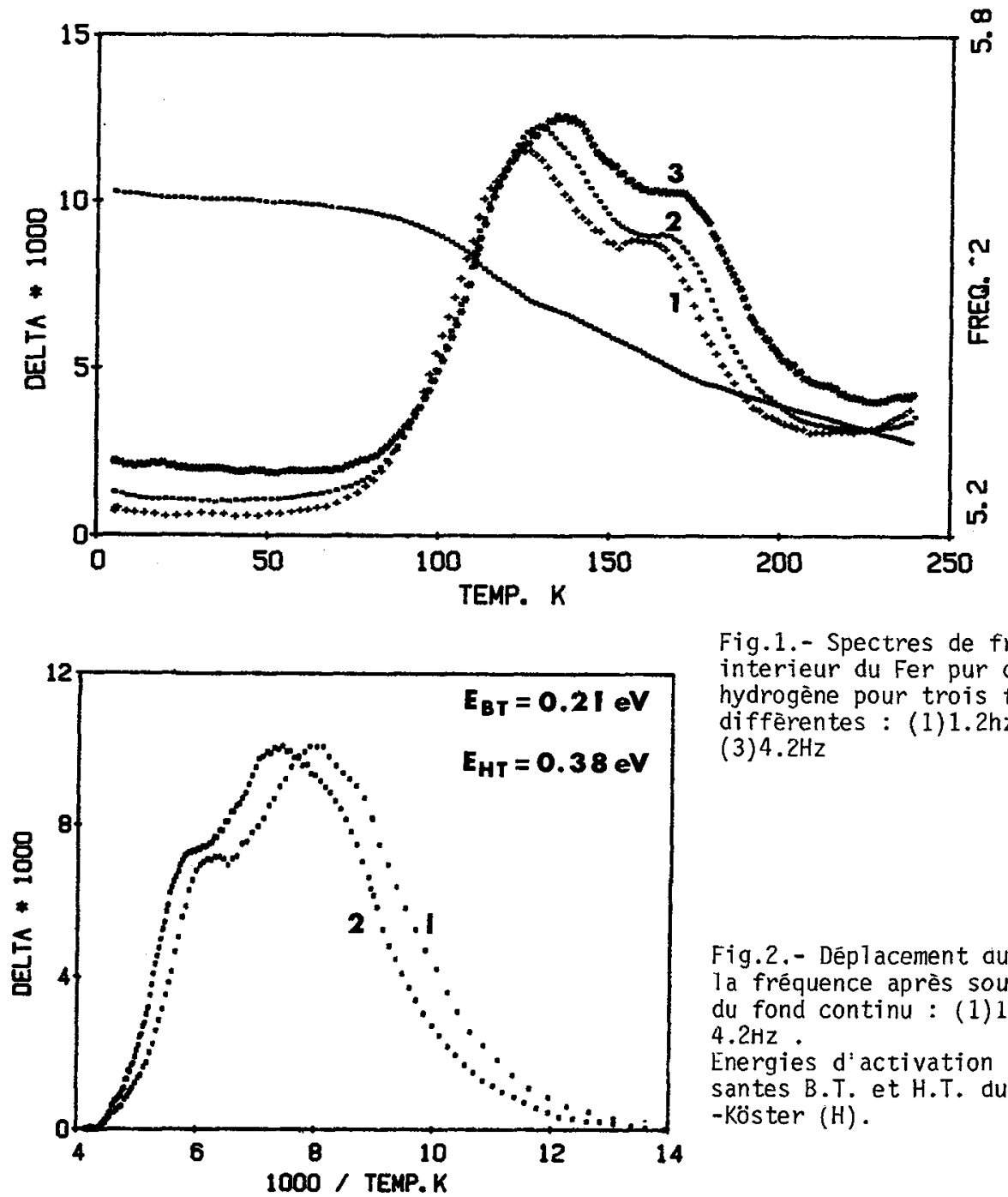

Fig.1. - Spectres de frottement interieur du Fer pur chargé en hydrogène pour trois frêquences diffërentes : (1) $1.2 \mathrm{hz} ;(2) 2.4 \mathrm{~Hz}$ (3) $4.2 \mathrm{~Hz}$

Fig.2.- Déplacement au pic avec la fréquence après soustraction du fond continu : (1) $1.2 \mathrm{~Hz}$; (2) 4. $2 \mathrm{~Hz}$. Energies diactivation des compo santes B.T. et H.T. du pic Snoek -Köster $(H)$. 


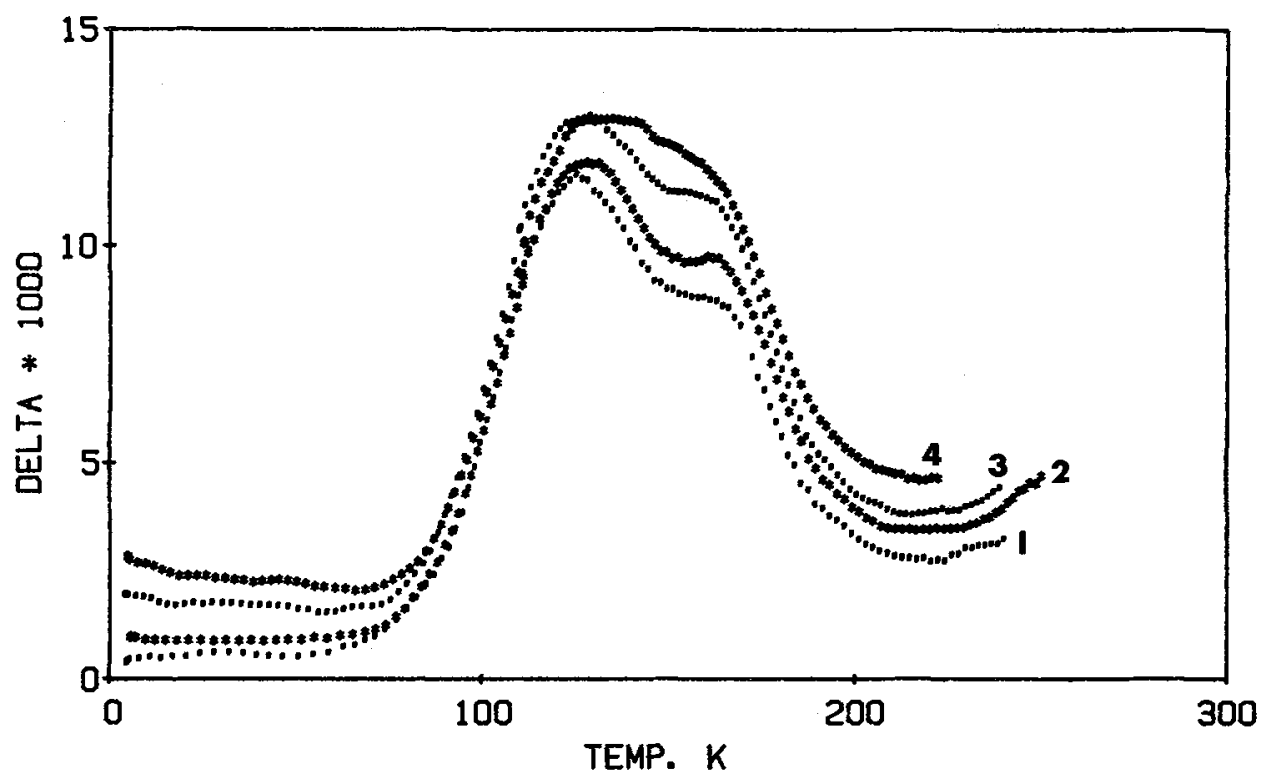

Fig.3. - Spectres de frottement intérieur pour differentes amplitudes de soliticitation $\varepsilon_{\mathrm{m}}$ : (1) $2.5 \times 10^{-6}$; (2) $1 \times 10^{-5} ;(3) 2 \times 10^{-5} ;(4) 3.8 \times 10^{-5}$

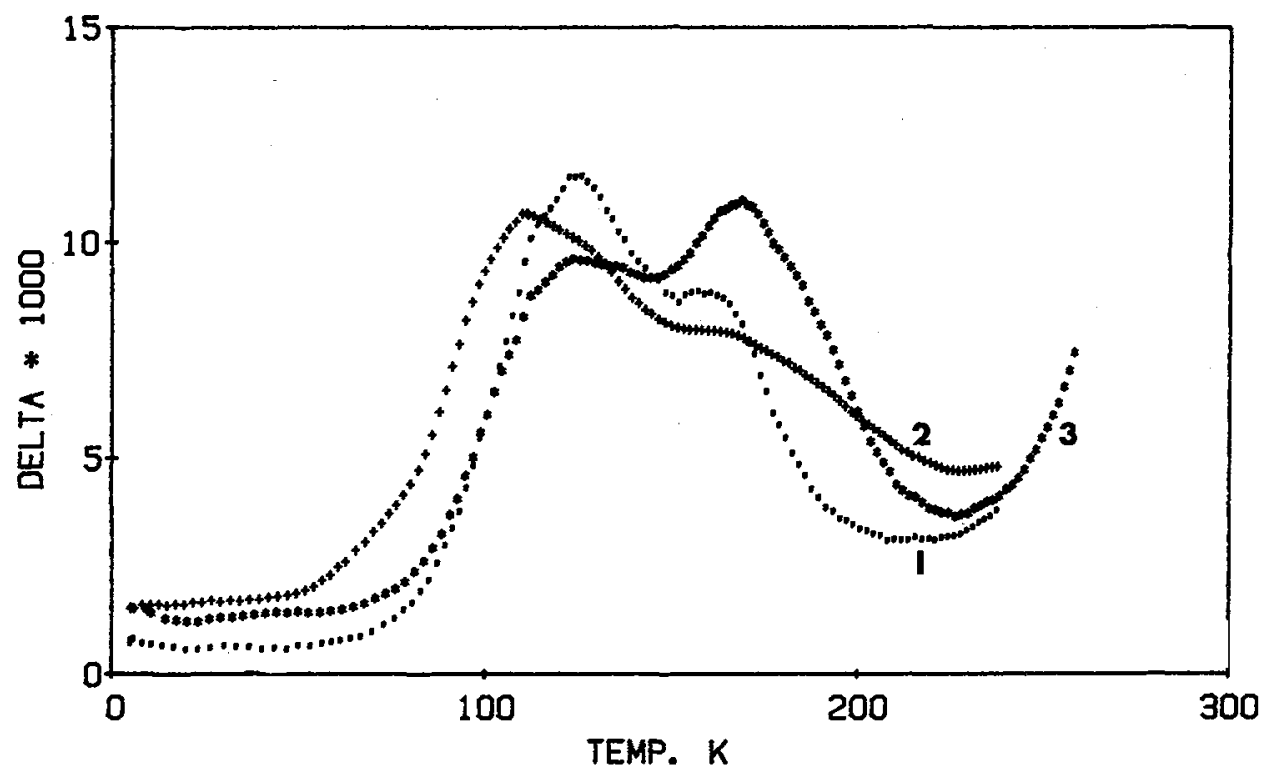

Fig.4.- Influence d'une déformation basse température sur le spectre de frottement interieur : (1) Avant déformation; (2) après déformation à $85 \mathrm{~K}$; (3) après rëcuit à $240 \mathrm{~K}$. 


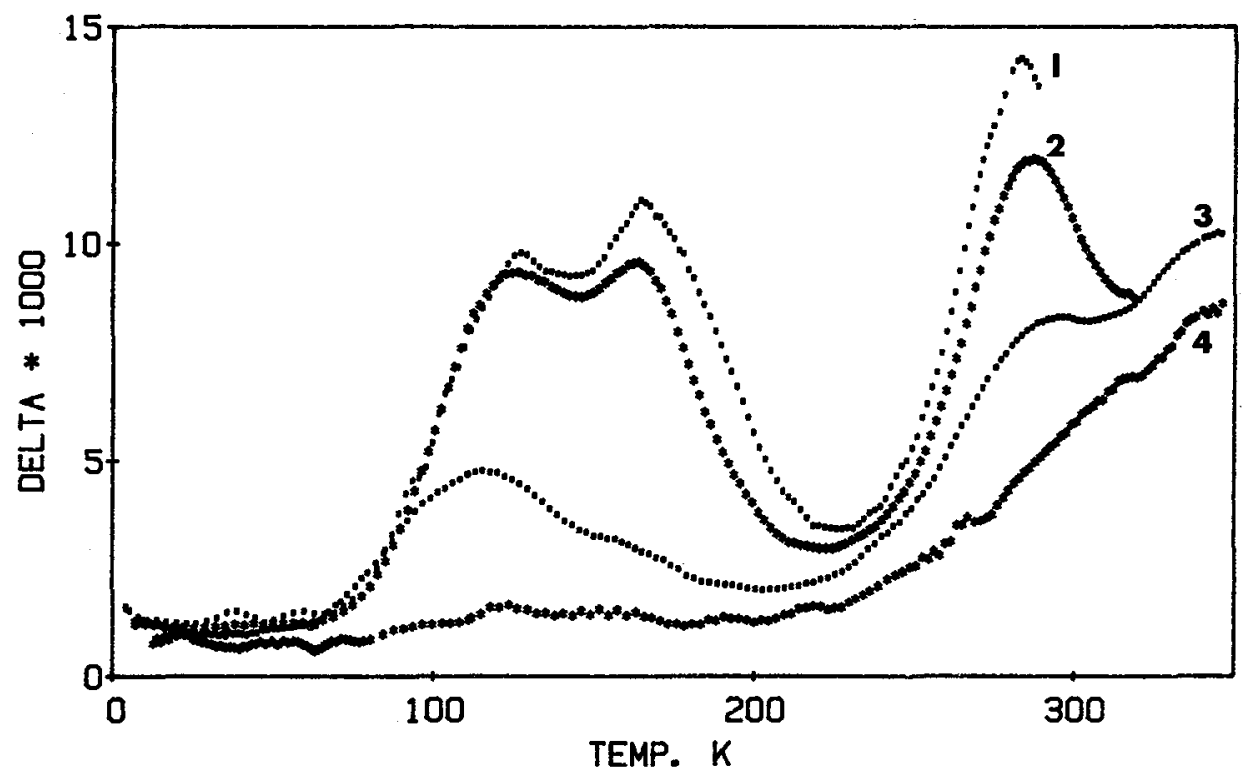

Fig.5.- Evolution du spectre de frottement interieur avec la tempera ture de récuit. Après récuit à : (1) $260 \mathrm{~K}$; (2) $290 \mathrm{~K}$; (3) $320 \mathrm{~K}$; (4) $350 \mathrm{~K}$ -

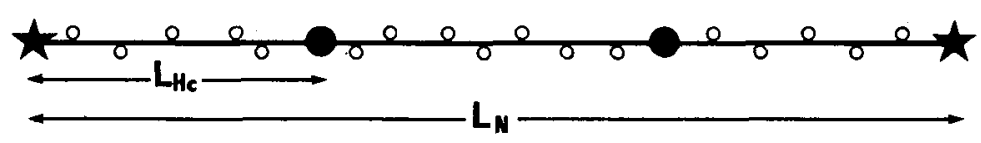

B.T.

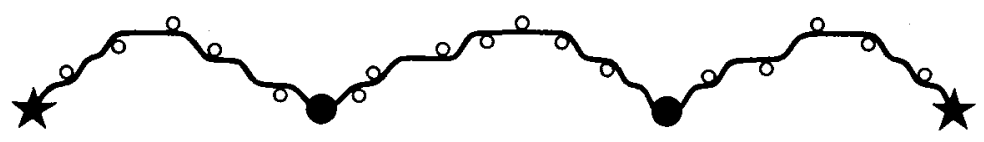

H.T.

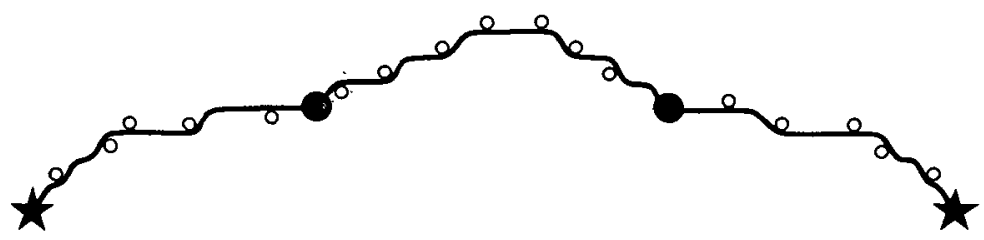

Fig.6.- Modèle d'interaction dislocation-nydrogène cans le fer pour le pic Snoek-Köster $(H)$.

o a tome d'hydrogène ; amas d'hydrogène 